\title{
ПРОБЛЕМА СГОВОРА УЧАСТНИКОВ ТОРГОВ И ПУТИ ЕЕ РЕШЕНИЯ В РАМКАХ ПАРАДИГМЫ ЦИФРОВОЙ ЭКОНОМИКИ С ПРИМЕНЕНИЕМ КВАНТОВОЙ ТЕОРИИ ВЕРОЯТНОСТЕЙ
}

\section{THE PROBLEM OF COLLUSION OF BIDDERS AND WAYS TO SOLVE IT IN THE FRAMEWORK OF DIGITAL ECONOMICS PARADIGMS AND BY USING QUANTUM PROBABILITY THEORY}

R. Iuryev

A. Alodjants

Summary. The work is devoted to the description of new ways of the decision of problems of automation of legal processes in the framework of current problems of setting up of methods of an artificial intellect in law enforcement practice in the conditions of digitization. The current conditions for the development of economics and technology require detailed regulation of the application of artificial intelligence algorithms, including at the legislative level, and in some cases this regulation at the level of regulations is still insufficient. The paper proposes a new approach to solving the problem of recognition and evaluation of collusions of two (and more) participants in public procurement, based on the theory of quantum information. The mathematical criterion of quantum entanglement revealed the degree of consistency in the behaviour of bidders in public tenders, confirmed by available data and expert evaluation. We develop software based on real data for the task of collusions detection; the results were tested by means of expert evaluation of the results.

Keywords: automation of legal processes, artificial intelligence, quantum information, consistency of behavior.

\author{
Юрьев Родион Николаевич \\ Университет ИТМО, г. Санкт-Петербург \\ juryev7@gmail.com \\ Алоджани Александр Павлович \\ Д.ф.-м.н., профессор, Национальный Центр \\ Когнитивных разработок; Университет ИТМО, \\ 2. Санкт-Петербург \\ 1. Аннотачия
}

Аннотация. Работа посвящена описанию новых способов решения задач автоматизации юридических процессов в свете текущих проблем внедрения методов искусственного интеллекта в правоприменительной практике в условиях цифровизации. Текущие условия развития экономики и техники требуют детального регулирования вопросов применения алгоритмов искусственного интеллекта, в том числе на законодательном уровне, и в ряде случаев это регулирование на уровне нормативных актов является ещё недостаточным. В работе предложен новый подход для решения задачи распознавания и оценки сговоров двух (и более) участников государственных закупок, основанный на теории квантовой информации. Математический критерий квантовой запутанности позволил выявить степени согласованности поведения участников закупок на публичных торгах, подтвержденный имеющимися данными и экспертной оценкой. На основе реальных данных разработан прототип программного обеспечения для реализации задачи выявления сговоров, проведена апробация результатов на базе экспертной оценки результатов.

Ключевые слова: автоматизация юридических процессов, искусственный интеллект, квантовая информация, согласованность поведения.

не только стимулом для развития экономики, но и благодатной почвой для множества злоупотреблений. Недобросовестные игроки на бирже в состоянии микроскопическими операциями на формально законных основаниях обрушить экономику целой страны. Перевод на незначительную для жителя европейской страны сумму в адрес какого-нибудь приверженца террористической идеологии может позволить провести полноценный террористический акт с многочисленными жертвами. Мир стал слишком хрупким для того, чтобы позволить манипулировать им только на основе заранее прописанных правил, которые относительно злоумышленники легко обходят. В результате законо- 
дателю приходится делать нелегкий выбор, и всё чаще ему приходится устанавливать каучуковые нормы, передавая полномочия по оценке применимости нормы на уровень исполнителей. В качестве примера можно привести Методические рекомендации Банка России от 21 июля 2017 г. № 18-МР «О подходах к управлению кредитными организациями риском легализации (отмывания) доходов, полученных преступным путем, и финансирования терроризма», где, по сути предлагается осуществлять комплексную оценку всех критериев сомнительных операций, указывается на то, что сами по себе ни неуплата налогов, ни переводы по счетам, не могут являться однозначными критериями операций по отмыванию. С учётом того, что денежные переводы в настоящее время проводятся в режиме реального времени, то есть задержки в перечислении составляют секунды, а количество проводимых банковских операций растёт с каждым годом, становится ясно, что по своей сути эти методические рекомендации обращены не к сотрудникам банка, которые должны контролировать поведение клиентов, а к разработчикам программного обеспечения для оценки рисков. Это - очень существенный момент, который мы можем недооценивать, так как всё происходит на наших глазах, и мы сами являемся участниками этого процесса, из-за чего оценка происходящего может искажаться. Но мы уже находимся в той реальности, когда законодательство пишется не для правоприменителей, а для разработчиков программного обеспечения. Конечно, формально никто не отказывается от ведущей роли человека в принятии решений на основе той информации, которую выдаёт программа, но в реальности уже подразумевается, что большинство операций должно осуществляться программой без участия человека. Более того нелинейный (экспоненциальный) рост информации присущий современному обществу (см. напр. Ahmed K. Noor, Potential of Cognitive Computing and Cognitive Systems, Open Eng. 2015; 5:75-88), и связанный с банковскими онлайн операциями требует адекватного подхода как в организации самого процесса, так и верификации установленных процедур. Банки, обязанные соблюдать все требования по борьбе с отмыванием средств, просто не в состоянии содержать такой штат в службе безопасности, чтобы осуществлять принятие решений о блокировке сомнительных операций в ручном режиме, не говоря уже об экономической составляющей в виде себестоимости ручного труда ради отслеживания операций на 10000 рублей.

Возможным решением данной проблемы является цифровизация, которой предстоит проникнуть во все стороны общественной жизни страны, экономики, финансов, что следует из Национальной стратегии развития искусственного интеллекта на период до 2030 года (утв. Указом Президента РФ от 10 октября 2019 г. № 490).

Несмотря на все возрастающую и прогрессивную роль процесса цифровизации, он обладает также и определенными издержками, связанными с необходимостью математической формализации задач, которые до сих пор лежали в гуманитарной плоскости своего решения. Политическая и общественная оценка происходящего лежит за пределами нашей статьи, однако отметим, что во всём мире эти процессы получают как положительные, так и негативные оценки со стороны общества. Противники цифровизации обращают внимание на нарушение прав личности при тотальном контроле и почти повсеместной прозрачности, сторонники подчёркивают удобство быстрых операций, перетока капиталов и т.д.

В настоящее время не только в России, но и в других странах обострилась проблема блокировки счетов компаний в связи с подозрением в отмывании денег. Блокировки может быть оспорены в судах, однако борьба с довольно сильным противником в виде банка может обходиться недёшево для компании, тем более, что банки вынуждены выплачивать проценты за оспоренные блокировки, как, например, в деле, рассмотренном Арбитражным судом Волго-Вятского округа, где банк заблокировал счёт клиенту, не удостоверившись в наличии оснований для блокировки в соответствии с действующим законодательством. Суд взыскал с банка проценты за пользование денежными средствами за весь период блокировки и обязал разблокировать счёт.

Это значит, что общие правила, установленные нормативными актами, были внедрены компанией неверно. Программное обеспечение, которым они воспользовались, выдало ошибочный ответ, который и привёл к убыткам для них и для их клиентов, а отдельно возможна ещё и ответственность перед надзорными органами. Но каким образом банк мог бы обезопасить себя от таких последствий? В первую очередь это предполагает запрос на правовую определённость нормативных актов, возможность предсказуемого ведения бизнеса для всех добросовестных участников экономической деятельности. В условиях, когда в нормативных актах приводятся только общие указания, причём, как мы рассматривали выше, указания для разработчиков программного обеспечения, обеспечить строгую правовую определённость невозможно и вот почему.

Перед разработчиком программного обеспечения стоит задача собрать воедино требования Центрального Банка РФ для распознавания сомнительной операции. Набор этих критериев должен быть преобразован 


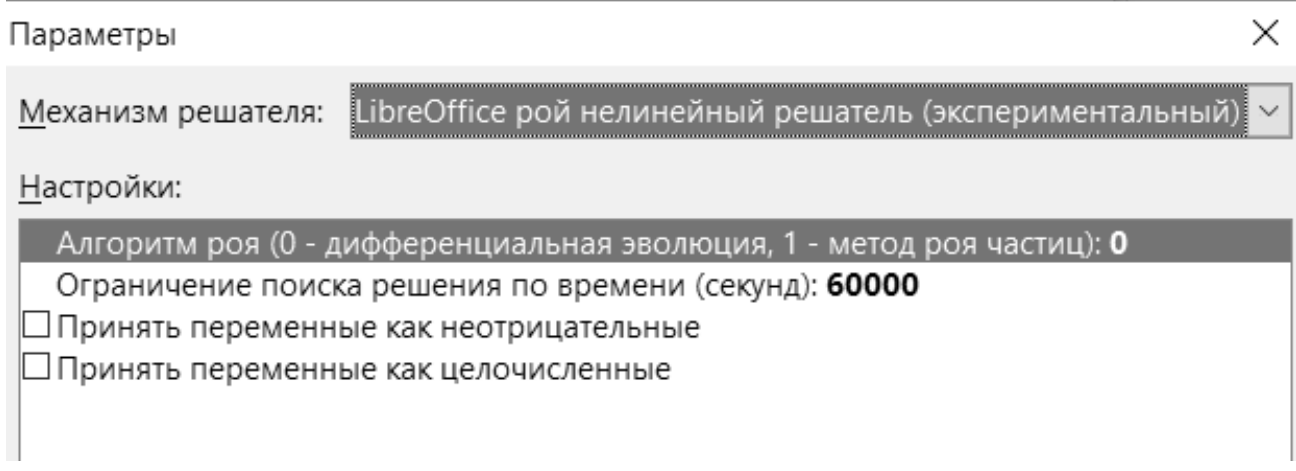

Рис. 1. Механизм решателя в LibreOffice.

из словесных инструкций в математически выверенный алгоритм для компьютера.

Например, такой критерий «фонд заработной платы сотрудников клиента установлен из расчета ниже официального прожиточного минимума» означает, что должна быть реализована выгрузка официальных данных о размере прожиточного минимума, вычислена сумма операций по зарплатному проекту клиента, разделена на количество сотрудников клиента и произведено сравнение с официальным прожиточным минимумом. Однако даже в таком несложном критерии у вдумчивого разработчика должны возникнуть вопросы. Правильно ли мы берём в качестве «фонда заработной платы сотрудников» сумму операций по зарплатному проекту? Или в неё нужно включать среднемесячный размер операций по зарплатному проекту? За какой период? Следует ли учитывать разные размеры заработных плат у сотрудников? Что нужно брать за основу: среднее арифметическое, медиану, моду?

Несложно показать, что в данном случае возможны разные варианты. Например, заработная плата 100000 рублей у руководителя и 5000 рублей у 10 сотрудников. В среднем заработная плата у 11 человек составляет 150000 рублей, то есть 13636,36 рублей на человека, что выше 11731 рублей, прожиточного минимума для трудоспособного населения за I квартал 2020 года согласно данным Федеральной службы государственной статистики. Однако если мы возьмём в качестве показателя медиану или моду значений, они составят 5000 рублей, что значительно ниже минимума.

В случае рассмотрения дела в суде может встать вопрос о том, каким образом рассчитывать эти суммы, и для каждого из вариантов можно найти обоснование, поэтому без конкретного указания со стороны законодателя никогда не будет определённости, а значит, не будет и уверенности у банка в том, что его не привлекут к ответственности за нарушения.
При этом многообразие математических методов в настоящее время таково, что специалисты в одной сфере математики не понимают специалистов в другой, не говоря уже о традиционном противостоянии между разными направлениями.

Выше мы рассмотрели только варианты с вычислением простого значения, но мы без труда можем отыскать в законодательстве более сложные вычисления. Например, п. 3.13 Правил формирования страховых резервов по страхованию жизни устанавливает, что «величина математического резерва на дату расчета, не совпадающую со страховой годовщиной, рассчитывается методом интерполяции через величины математического резерва на ближайшие к дате расчета страховые годовщины», а п. 2.1 ст. 6 Федерального закона от 21.12.2013 № 353-Ф3 «О потребительском кредите (займе)» и вовсе содержит пугающую формулу расчёта процентной ставки базового периода (i):

$$
\sum_{k=1}^{m} \frac{\text { дП }_{k}}{\left(1+e_{k} i\right)(1+i)^{q_{k}}}=0
$$

Стоит отметить, что вычисление этой ставки без использования компьютера является нетривиальной задачей, потому что закон (вслед за экономической теорией) предусматривает, что процентная ставка есть наименьшее положительное решение этого уравнения. За этими скупыми словами стоит целая отрасль математики, ставящая своей задачей поиск экстремума функции, использующая множество методов и приёмов, которые могут различаться по точности. Понятно, что встроенные в электронных таблицах программы поиска этого значения имеют некоторое приближение (кстати, в открытом редакторе электронных таблиц Calc из пакета LibreOffice некоторые функции помечены как «экспериментальные», так как находятся в стадии разработки и создатели не гарантируют точный результат). 
Итак, при формулировании норм законодатель в наше время обязан задумываться о том, как эти нормы будут реализованы в программном обеспечении, а для этого нужно соблюдать несколько правил.

В первую очередь необходимо чётко указывать на используемые параметры для расчётов. С этой точки зрения указание на соотношение между «размером фонда заработной платы» и размером официального прожиточного минимума не отвечает принципам правовой определённости и недопустимо в нормативных актах.

Затем законодатель должен определиться с тем, какие средства расчётов являются эталонными для проведения ответственных вычислений. Без ясного понимания степени отклонения в вычислениях от заданной точности мы не можем быть уверенными в результате и справедливости применяемых мер. Достаточно здесь вспомнить о понятии «значительного, крупного и особо крупного размера» наркотических средств, где от вычисления пропорции вещества в смеси (0,0001 грамма для ацетрофина) зависит оценка тяжести связанного с этим преступления. Между тем компьютер может оперировать только такими числами, точность которых конечна, при этом разные языки программирования содержат разные подходы к определению конечной точности дробных чисел (или чисел с плавающей точкой). Это означает, что мы обязаны учитывать ещё и эти обстоятельства.

Bсе, обозначенные выше примеры касаются технических вопросов и могут быть в принципе решены путём стандартизации. Более сложным является вопрос о фундаментальных ограничениях, связанных с развитием искусственного интеллекта в решениях поставленных задач.

Надо заметить, что граница между языком программирования и текстом технического задания (а со временем, можно предполагать, и нормативным актом) становится всё менее заметной. В некоторых языках программирования инструкции уже достигли очень высокой степени соответствия с естественным языком, например, в языке SQL инструкции пишутся почти «человеческим» языком: «INSERT 'текст` INTO `таблица' WHERE `номер` = 1 ».

Если обращаться к многочисленным определениям «искусственного интеллекта», то мы обнаружим, что к нему относятся системы, которые думают либо действуют подобно людям и те, которые делают это рационально. В первом случае речь идет об антропоморфных системах, которые наряду с важными (когнитивными) функциями человека могут принимать нера- циональные решения. В этой связи возникает важный вопрос - каким образом такие решения могут приводить к отрицательному результату.

С другой стороны, система, которая руководствуется только рациональными решениями (например, обычные ЭВМ с заложенных в них булевской логикой), очевидно, не является искусственным интеллектом; она всего лишь способна проверить соответствие определённого поля определённому числовому значению, что, по сути, бессмысленно, и именно это, по нашему практическому опыту, и представляет основную проблему старой парадигмы «экспертных систем». Дело в том, что все они составлялись на основе экспертного или, правильнее было бы назвать, порогового подхода, когда эксперт устанавливал некоторые пороги значений, относительно которых и оценивалось состояние исследуемого объекта. При несоответствии объекта пороговому значению сообщалось об отклонении. Системы, построенные по такому принципу и содержащие множество параметров для разных измеряемых состояний, зачастую оказываются бесполезными из-за большого количества ложных срабатываний при работе с реальными объектами. Грубо говоря, такая система будет непрерывно сигнализировать о наличии нарушений во всех финансовых операциях любого, даже самого добросовестного клиента банка.

Сложно сказать, после какого момента начинается обессмысливание процесса пороговой оценки объекта в общественных отношениях, хотя это - интересная тема, касающаяся очень многих сфер и требующая отдельного изучения. Пример с размером наркотиков является наиболее наглядным, ведь, по сути, законодатель имеет очевидное намерение установить разную ответственность не за вес как таковой, а за разные дозы для личного потребления и для продажи. Не имея никакого иного инструмента кроме пороговой оценки, законодатель вынужден использовать именно его, так как в противном случае он должен был бы отдать определение этой дозы на уровень правоприменителей, что может вызывать злоупотребления и сомнения в объективности.

Именно проблема многочисленных ложных срабатываний (или, как это называется в науке о данных, «ошибок первого рода») является одним из основных препятствий в развитии систем искусственного интеллекта. Однако с развитием новых методов искусственного интеллекта, основанных на нейронных сетях, эта программа становится более «умной» и принимает решение на основе множества факторов так же, как это сделал бы человек. Успехи нейронных сетей в распознавании изображений широко известны и в профессиональной среде уже рассматриваются как нечто само собой разумеющееся. Совсем недавно программа для 
распознавания текста Fine Reader казалась чем-то невероятным, а сейчас такую программу делают студенты на вводных лекциях по искусственному интеллекту; на улицах мы уже можем видеть беспилотные автомобили, в местах боевых действий - беспилотные летательные аппараты, и т.д. На данный момент уже нет серьёзных препятствий к тому, чтобы поручать решение ряда юридически значимых вопросов нейронным сетям, более того, это активно практикуется в ведущих компаниях. Например, СБЕР сообщает об использовании технологии глубокого обучения в задачах выявления банковского мошенничества. На данном этапе решение о наличии признаков преступления не принимается исключительно программой, она лишь предоставляет информацию для принятия решения человеку и не за горами тот момент, когда нейронная сеть будет распознавать поведение точнее, чем любой специалист службы безопасности, не говоря уже о том, что она в состоянии осуществлять отслеживание в режиме реального времени, практически в момент совершения транзакции - возможность, которая исключена для человека в принципе. Конечно, новая реальность заставит нас учитывать и новые риски, например, такие явления как Deep Fake, когда нейронные сети пытаются обмануть с помощью других нейронных сетей.

\section{Новый этап развития \\ законолательства - регулирование алгоритмов принятия решений}

Таким образом, мы приходим к новому повороту в регулировании общественных отношений. На одном этапе законодатель имел возможность устанавливать общие положения, давать указания правоприменителю в расчёте на его правосознание («Имея в виду, что законом не установлены критерии отнесения находящихся в незаконном обороте наркотических средств или психотропных веществ к небольшому, крупному, особо крупному размеру, этот вопрос должен решаться судом в каждом конкретном случае исходя из их количества, свойств, степени воздействия на организм человека, других обстоятельств дела...»,- так предлагал Верховный Суд РФ решать проблему неясности ранее действовавшей ст. 224 УК РСФСР).

На следующем этапе законодатель снабдил нас подробнейшими сведениями о микрограммах наркотиков с точными градациями, исключающими любое усмотрение суда, зато вызывающими сомнение в справедливости, так как согласно исследованиям, наибольшее количество приговоров приходится именно ровно на пограничный размер. Такие скачки от расширения судейского усмотрения к его сужению не являются исключительно российским явлением и характерны и для других стран, и для других эпох.
Но, очевидно, в настоящее время мы подходим к следующему, принципиально новому этапу, когда решение по некоторым вопросам будет передаваться полностью (или частично) искусственному интеллекту, который будет осуществлять эту оценку на основе обработки сотен тысяч образцов. Отчасти, в этой системе уже существуют и работают все клиенты высокотехнологичных банков, подобных Сбербанку или Банку Тинькофф, но впереди всё больше подобных примеров. Распознавание содержимого грузов на таможне, выявление подозрительных лиц на улице, распознавание нарушения правил дорожного движения,- это всё уже существует сейчас, и законодателю бессмысленно закрывать глаза на то, что решения здесь уже принимает нейронная сеть, а не человек.

Что же предлагается урегулировать в этой сфере? Для начала необходимо осознать проблему в комплексе, так как у неё есть очень много сторон: от нарушения прав человека до чисто технических проблем. Затем необходимо создавать каталог требований к техническим решениям для использования на практике правоприменителями и правила их использования. Если речь идёт о блокировке счёта в связи с совершением сомнительной операции, общество, банк, клиент, имеют право по крайней мере понимать причины такой блокировки. К сожалению, нейронные сети в большинстве случаев ответ на такой вопрос дать не в состоянии. Тем не менее, при должной постановке задачи наиболее значимые характеристики можно выявить и проконтролировать, например, с помощью автоматической проверки со стороны Центрального Банка РФ или Росфинмониторинга. То же самое будет касаться и беспилотных автомобилей, для которых жизненно необходимо устанавливать критерии допуска на улицу (впрочем, стандарты для них уже активно разрабатываются).

В настоящей работе рассматривается принципиально новый подход к проблеме выявления сговора в совершенно традиционной сфере, затрагивающей большинство предпринимателей, - публичных торгах, и основанный на применении квантовоподобных алгоритмов к задачам принятия решения. В отличие от тех же алгоритмов глубокого обучения для искусственного интеллекта, применение квантовой теории вероятностей здесь становится оправданным ввиду относительной простоты реализации алгоритма и его последующей интерпретации.

\section{Проблемы организации публичных закупок в России и зарубежом}

Выявление сговоров является крайне актуальной задачей как в РФ, так и за ее пределами. Согласно разным подсчётам, из-за сговоров теряется до $20 \%$ общего 
бюджета Европейского Союза. Подсчёты применительно к России дают оценочные объёмы потерь в районе 1-2 триллионов рублей ежегодно.

Статья 24.1 Федерального закона «О контрактной системе в сфере закупок товаров, работ, услуг для обеспечения государственных и муниципальных нужд» от 05.04.2013 № 44-Ф3 предусматривает осуществление всех закупок в Российской Федерации путём проведения аукционов либо конкурсов. При этом все торги размещаются на едином электронном ресурсе - сайте https://zakupki.gov.ru/epz/main/public/home.html.

В 2020 году экономия при заключении контрактов по всей России составила 228,67 миллиардов рублей при общем цене контрактов 4,7 триллиона рублей. Аналогичные схемы госзакупок реализованы в EC (https:// ted.europa.eu/TED/main/HomePage.do) и в других странах. Министерство экономического развития РФ ведёт мониторинг зарубежных и российских порталов публичных закупок на своём сайте.

Система государственных закупок является существенным инструментом государственного управления, обеспечивающим прозрачность процедур распределения бюджетных средств, планирования, статистического учёта, определения реальных цен на рынке и т.п. Бесперебойно работающая система закупок для государственных нужд является неотъемлемой частью механизма управления любого современного государства.

Естественно, всегда существуют лица, заинтересованные в обмане системы. В первую очередь этим занимаются недобросовестные поставщики в связке с организаторами государственных закупок, задача которых - устранить конкурентов, сделать торги безальтернативными и дать возможность победить только одному, заранее определённому участнику.

Механизм такого обмана давно известен, в первую очередь это такая формулировка требований к предмету поставки, что под неё будет подходить только один единственный участник. Из-за этого объём закупочной документации на какие-нибудь канцелярские товары раздувается до 200-страничных договоров, с подробнейшими спецификациями товара и жесткими условиями сделок.

Но, на пути к успешному обману системы торгов есть одно ограничение, мешающее преступникам довести дело до конца втайне: как бы они ни исхитрялись, если в торгах примет участие только один участник, они будут признаны несостоявшимися, и в результате всё сорвётся либо потребует соблюдения специальных процедур (в РФ необходимо получение согласования сделки с Управлением ФАС). Чтобы такого не случилось, в торгах всегда принимает участие другой, подставной участник, за счёт которого придаётся видимость законности процедуре торгов и скрывается реальный сговор между госзаказчиком и поставщиком.

Конечно, давно ушли в прошлое такие схемы, при которых участники сговора возглавлялись одним и тем же директором, имели одинаковый адрес и одинаковых учредителей. Такие схемы сразу привлекают внимание и легко вскрываются. Поэтому правонарушители стараются скрывать связи между собой, используют разных учредителей, разные адреса, заходят на торги с разных компьютеров (IР-адресов). Однако есть всё-таки одна вещь, которой они не могут избежать,- - это похожесть поведения, выражающаяся в некоторой согласованности в различных ситуациях (контекстах принятия решения). Как бы ни пытались они скрывать свою взаимосвязь, они остаются участниками договорённости действовать совместно, имеют общую цель, общие инструменты достижения этой цели, согласовывают своё поведение и в результате выдают себя именно им. Выявление уже самих этих контекстов представляет само по себе нетривиальную задачу.

\section{Механизм распознавания сговора на основе квантовой теории вероятностей}

В отличии от классической теории вероятностей (лежащей в основе алгоритмов глубокого машинного обучения), в квантовой теории определенный контекст (условия) измерения являются ключевым фактором, влияющим на конечный результат. Важно, что здесь мы имеем ввиду вовсе не распознавание сговора с помощью так называемого квантового компьютера, а применение квантовой математики со своей «философией» измерений за пределами естественных наук.

В своё время вопросы распознавания характеристик человека были глубоко разработаны в рамках такого направления как бихевиоризм. Несмотря на значительные недостатки этого направления в психологии, достижения учёных того периода являются полезными с точки зрения распознавания скрытых взаимосвязей между исследуемыми объектами, особенно в ситуации, когда других источников данных на этом этапе исследования у нас нет и не может быть. Но для того, чтобы по поведению, то есть по внешне проявляемым признакам внутреннего процесса, определять сам процесс, состояние или иные характеристики исследуемого объекта, необходимы зачастую непростые вычисления, основанные на классических вероятностных методах и подходах в анализе сложных схем процессов принятия решений, 
что в результате и позволяет восстановить исходную картину по последствиям. Решение такого рода задач всегда представляет значительные трудности, поскольку требует учитывать множество факторов и их взаимосвязей. Проще говоря, здесь речь идет о анализе принятия решений в условиях неопределенности, что в некоторых случаях может оказаться невыполнимой задачей.

Подходы квантовой теории вероятностей принципиально отличны. Они основаны на том факте, что эти неопределенности в априорно существуют и сопутствуют квантовой системе при ее эволюции и измерениях, проводимых с ее участием. С недавних пор квантовый аппарат стал успешно применяться для описания систем за пределами естественных наук - в психологии, социологии. Заметим, что когда говорится о квантовых вычислениях, это совершенно не означает, что речь идёт о какой-либо «квантовой природе» социальных или психических явлений. Нельзя рассматривать всерьёз маргинальные теории, пытающиеся чуть ли не объяснить человеческую психику причинами, лежащими в основе квантовой теории. Об этом приходится напоминать, поскольку в популярной литературе фигурируют такие понятия как «квантовое сознание», «квантовая психология» и прочие ненаучные концепции. Основой таких концепций выступают работы Р. Пенроуза и некоторых других американских учёных. В их работах постулируется некая возможность «квантового воздействия с помощью мысли» на объект, основанная на физических проблемах измерений в квантовой теории. Следует согласиться с теми авторами, которые относят такого рода концепции к маргинальной парапсихологии, не имеющей ничего общего с научными работами. Конечно, нельзя говорить и о какой-либо «теории всего», якобы вытекающей из применения квантовых принципов в сфере гуманитарных знаний.

Достоинством подхода квантовой теории является возможность быстрого и удобного оперирования со множеством потенциальных состояний системы, определенных в Гильбертовом пространстве и имеющих вероятностную интерпретацию в части своей реализации в зависимости от контекста измерения. Именно существование контекстно зависимых измерений (т.е. измерений зависящих от условий их реализации) делает квантовый подход весьма полезным в психологии принятия решений. В частности, применительно к экономическим и социогуманитарным исследованиям известны случаи успешного применения математического аппарата квантовой теории для решения таких задач как предсказание иррационального поведения потребителей на рынке (Канеман, Тверски, Шафир). Вопреки старому традиционному представлению о потребителе на рынке как рациональном субъекте, который делает выбор согласно рациональным представлениям и предпосылкам, экономическая теория показывает невозможность предсказания в рамках классической теории. Тривиальный вывод о том, что покупатель в магазине действует совсем не рационально, а даже иногда вопреки рациональным предположениям, потребовал применения к задаче предсказания его поведения новых методов, которые не были доступны в рамках старой концепции и не могут быть применены без расширения математического аппарата. Оказалось, что единственным математическим аппаратом, пригодным для таких предсказаний, является аппарат квантовой теории. Главным открытием учёных в этой сфере было то, что поведение участников социальных, экономических и психологических экспериментов полностью описывалось с помощью квантовой теории независимо от различий языков, культур или социальных статусов.

\section{Метрика согласованности \\ (concurrence) $\Delta \wedge$ s выяв^ения \\ сговора на торгах}

В нашем исследовании к задаче выявления согласованного поведения участников государственных закупок был использован хорошо известный в квантовой теории критерий оценки особого состояния «запутанности» (entanglement) двух частиц. Не вдаваясь в несущественные для нас подробности квантовой теории, поясним, что две элементарные частицы, находясь в «запутанном» состоянии, можно сказать, «ведут себя» коррелировано (согласованно), даже находясь на большом расстоянии друг от друга и не коммуницируя между собой. Причиной такого поведения является тот факт, что изначально эти частицы были испущены одним и тем же источником частиц обладающим определенными свойствами. Например, молекула, имеющая спин равный нулю, распалась на два атома с противоположно направленными спинами, которые разлетелись в противоположных направлениях. Неопределенностью здесь является направленность спинов (внутреннего момента количества движения) атомов до измерения. Однако, если конкретное направление спина одной из частиц определено в результате измерения, то спин другой частицы - «автоматически» идентифицируется как противоположный.

Важной деталью здесь является тот факт, что для внешнего наблюдателя априорно может быть не известен тот факт, что частицы были испущены в результате распада одной молекулы. В этом случае необходимо провести несколько измерений, в результате которых мы сможем (с некоторой вероятностью) определить насколько скоррелированы состояния этих частиц. В квантовой теории для распознавания «запутанного» состояния у априорно неизвестных нам 
(как наблюдателю) частиц введен так называемый параметр согласованности $C$ (concurrence), который $C=\left|\left\langle\psi\left|\hat{\theta}_{y} \otimes \hat{\theta}_{y}\right| \psi\right\rangle\right|, 0 \leq C \leq 1$, где $\psi>-$ квантовое состояние двух частиц, $\hat{\sigma}_{y}-$ матрица Паули.

Таким образом, если $C=0$, то частицы полностью независимы. Наоборот, при $C=1$, полностью зависимы и их поведение согласовано. Значение $C$, полученное в пределах $0<C<1$ означает степень согласованности (запутанности) частиц.

\section{Метолика эксперимента и его моделирование}

В нашем случае алгоритм выявления «запутанности» частиц предложен для выявления согласованного поведения участников государственных закупок. Заметим, что алгоритм не отвечает на вопрос каким образом сговорились участники торгов (это нам и не интересно). Алгоритм выявляет определенные корреляции в их поведении, в действиях с учетом торгов относительно других участников торгов. Заметим, что сама по себе идея применения этого алгоритма за пределами собственно квантовой теории не нова. В литературе опубликованы положительные результаты его применения в сфере выявления семантических связей, однако В отношении публичных торгов алгоритм применяется впервые. Важно, что в отличие от квантовой физики - в социальных науках соответствующие алгоритмы, методы и подходы имеют не столько абсолютное, сколько относительное значение и смысл, в связи с чем нередко называются квантовоподобными. А именно, применение квантовоподобного алгоритма позволяет за счет использования квантовой математики и соответствующих критериев к обработке данных выявить определенные (превышающие некий «средний» уровень) корреляции в поведении агентов принятия решения, указывающие на их «аномальное» поведение. Отдельный интерес представляет то обстоятельство, что по итогам сравнения характеристик производительности оптимальным инструментом для вычислений оказалась библиотека квантовых вычислений Qiskit, paзработанная компанией IBM для квантового компьютера. В случае если этот квантовый компьютер окажется пригодным для практических задач, на нём можно будет решать задачи по отслеживанию сговоров на торгах. Следует заметить, что библиотеке Qiskit использован альтернативный, несколько отличный от традиционного способ вычисления параметра concurrence.

Для начала необходимо определиться с метриками для вычислений, иными словами: что мы будем считать за наличие согласованного поведения, а что - нет. Для этого была создана шкала, где максимальный балл давался в том случае, если участники торгов оказыва- лись замешанными в сговоре, и это было опубликовано в прессе либо было вынесено соответствующее решение территориального органа ФАС РФ. Минимальный балл давался в тех случаях, когда признаки согласованности были явно маловероятны (например, совершенно разные отрасли, разные регионы). Промежуточные варианты оставлены на усмотрение эксперта.

Следующий этап - сбор данных. Для выгрузки данных с сайта единой информационной системы в сфере государственных закупок предоставлен интерфейс для скачивания данных через FTP. Данные выложены по адресу: ftp://ftp.zakupki.gov.ru/. Логин и пароль для доступа к системе: free/free.

В корневом каталоге выбирается папка «3. fcs_ regions», содержащая данные полной выгрузки по регионам опубликованной сайте госзакупок информации.

В указанной папке выбирается каталог конкретного региона и внутри этого каталога выбирается папка «protocols».

В этом каталоге лежат заархивированные файлы XML, например, файл

«protocol_Zabajkalskij_

kraj_2014060100_2014070100_266.xml.zip», разме-

ром 1938 KB.

После скачивания этого файла его необходимо разархивировать. Внутри лежит много файлов. Нас интересуют не все из них, а только те, размер которых более 1 Кб и с расширением XML.

В качестве примера рассмотрим файл в каталоге «protocol_Zabajkalskij_

kraj_2014060100_2014070100_266.xml.zip»_-《fcsPro tocolOK1_0191300015614000035_29767.xml»

В этом файле нас интересуют следующие параметры:

$<$ purchaseNumber $>0191300015614000035$

$<$ /purchaseNumber $>$

Это число - является уникальным номером-идентификатором закупки. Номер уникален во всей информационной системе.

Далее необходимо проследовать по следующему пути узлах XML-документа:

$-<$ protocolLots $>$

$-<$ protocolLot $>$

-<applications $>$

-<application> 
Таблица 1

\begin{tabular}{|l|l|l|}
\hline Степень согласованности & Экспертная оценка & Обоснование \\
\hline 0,31897427243 & 0,75 & Сговор подтверждён ФАС \\
\hline 0,28020547364 & 1 & Учредитель замешан в доказанном сговоре \\
\hline 0,21810317681320202 & 0,75 & Сговор подтверждён ФАС \\
\hline 0,20471867031308802 & 0,75 & Сговор подтверждён ФАС \\
\hline
\end{tabular}

\section{Предсказанный результат вычисления согласованности относительно экспертной оценки сговора}

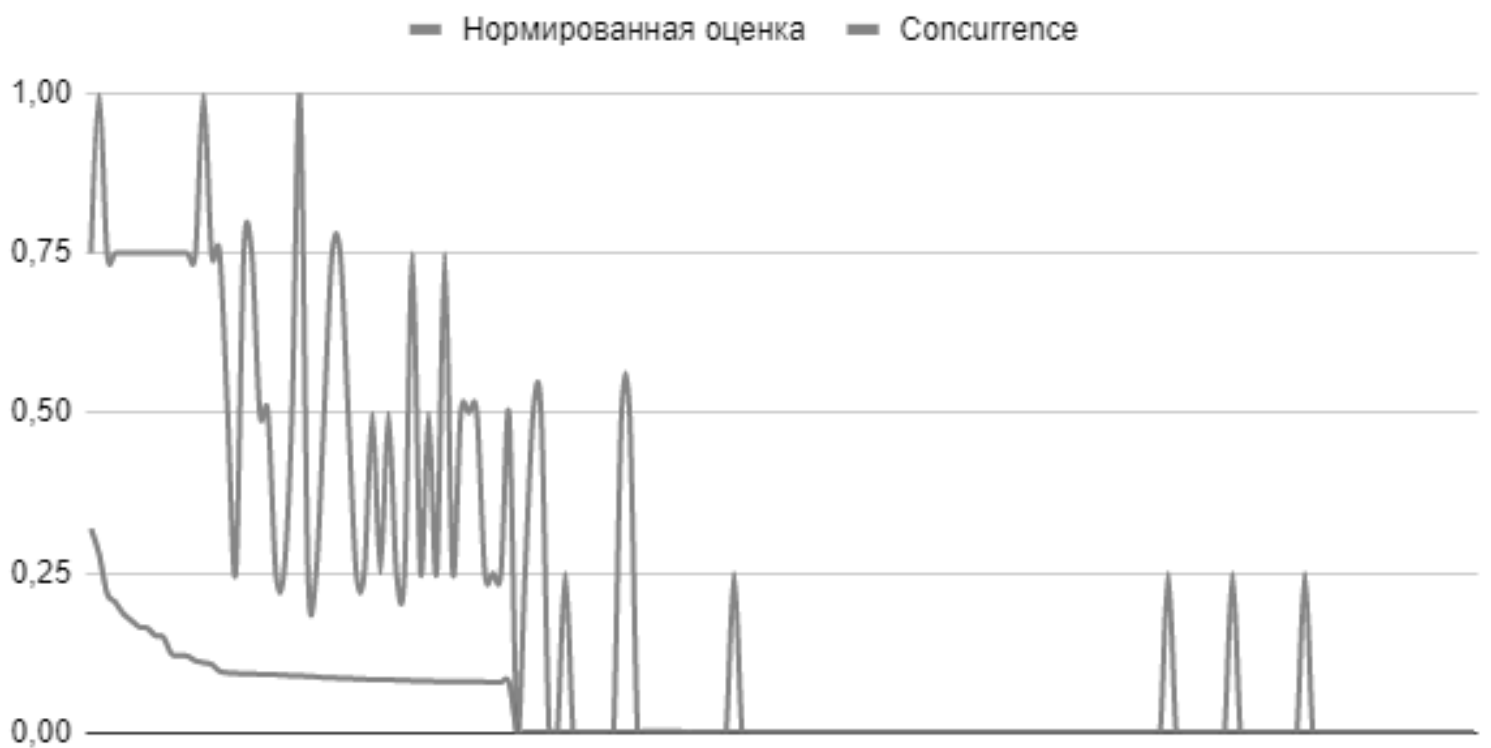

Рис. 2. Соотношение между параметром concurrence и нормированной экспертной оценкой

$<$ journalNumber $>1<$ /journalNumber $>$

<appDate>2014-05-26T08:30:00+10:00</appDate>

-<appParticipants>

-<appParticipant>

$<$ participantType $>\mathrm{U}<$ /participantType $>$

$<$ inn $>5027089703</$ inn $>$

Нас интересует последний - ИНН, который и представляет собой уникальный номер участника торгов, совпадающий с его индивидуальным налоговым номером.

Данные собираются в единую таблицу. Перед нами не стояла задача охватить всю территорию России и всех участников государственных закупок без исключения, поэтому в рамках исследования было охвачено около 6000 участников и около 7000 закупок нескольких регионах.

Воспользовавшись специализированным программным пакетом для обработки табличных данных Pandas, удалось достигнуть хороших показателей опти- мизации, так как в рамках работы на согласованность проверялся каждый участник с каждым участником.

Если наша гипотеза о применимости этого алгоритма к выявлению сговора верна, то должны быть получены следующие данные: чем выше параметр concurrence, тем выше вероятность наличия сговора между участниками. Оценка качества работы алгоритма осуществлялась экспертом (впрочем, как показано выше, в отношении верхней части таблицы его роль сводилась только к поиску упоминаний в прессе и в материалах УФАС информации о нарушителях).

Результаты оказались даже несколько неожиданными. Практически везде оценки эксперта совпадали с оценками программы.

Для оценки степени корреляции между оценками эксперта и результатами работы алгоритма применялся коэффициент корреляции Пирсона, который составил 0,81 . Но самое главное заключалось в том, что все 
участники торгов, получившие максимальные значения параметра concurrence, так или иначе подозревались или были признаны участниками сговора на госзакупках (в таблице 1 приведены первые 4 участника).

\section{Результаты исслеАования}

Таким образом, впервые была показана эффективность алгоритма вычисления параметра согласованности для решения вполне рутинной задачи государственного управления, с помощью которой можно автоматизировать процесс выявления сговоров на самых ранних стадиях, и, что особенно важно, по таким критериям, которые невозможно фальсифицировать или скрыть. Достигнутый результат является проверяемым, может быть оценён не только с точки зрения общих представлений о поведении участников, но и с помощью экспертной оценки. По сути, мы получаем в руки мощный инструмент, который, в отличие от аналогичных инструментов (например, нейронных сетей) отличается такой характеристикой как проверяемость.
Отметим, что нейронные сети здесь также могут быть использованы для выявления многостороннего сговора, но с применением квантовой математики. В этом случае речь, фактически идет, о новом направлении исследований с применением квантового машинного обучения, что находится в общем русле исследований в этой сфере. Хотя в представленном эксперименте речь идёт только об узкой сфере применения этого инструмента к выявлению только поведения на государственных закупках, это не значит, что его сфера ограничивается таким узким сегментом. Очевидно, что такое же поведение возможно и на любых публичных торгах, включая биржевые. Выявление такого рода согласованного поведения представляет особенный интерес в свете борьбы с финансовыми махинациями на рынке.

\section{Б^агоАарности}

Работа поддержана в рамках гос.задания 2019-1339 Министерства науки и высшего образования РФ.

\section{ЛИТЕРАТУРА}

1. Постановление Арбитражного суда Волго-Вятского округа от 25.12.2017 № Ф01-5062/2017 по делу № А43-36453/2016.

2. Федеральная служба государственной статистики. Величина прожиточного минимума. [Электронный ресурc] https://www.gks.ru/vpm (дата обращения: 25.7.2020).

3. Положение Банка России «0 правилах формирования страховых резервов по страхованию жизни» от 16.11.2016 № 557-П (Зарегистрировано в Минюсте России 29.12.2016 № 45055)// «Вестник Банка России», № 5, 19.01.2017.

4. Ван Хорн, Джемс К. С., Вахович, мл. Джон М. Основы финансового менеджмента: Пер. с англ.—СПб.: 000 «Диалектика», 2020.—С. 127.

5. Васильев Ф. П. Методы оптимизации: В 2-х кн.—Новое изд., перераб. и доп.—М.: МцНМ0, 2011.

6. Постановление Правительства РФ от 01.10.2012 № 1002 (ред. от 13.03.2020) «0б утверждении значительного, крупного и особо крупного размеров наркотических средств и психотропных веществ, а также значительного, крупного и особо крупного размеров для растений, содержащих наркотические средства или психотропные вещества, либо их частей, содержащих наркотические средства или психотропные вещества, для целей статей 228, 228.1, 229 и 229.1 Уголовного кодекса Российской Федерации»

7. Таненбаум Э., Остин Т. Архитектура компьютера. 6-е изд.-СПб.: Питер, 2015.—C. 708.

8. Рассел С., Норвиг П. Искусственный интеллект: современный подход. 2-е изд.: Пер. с англ.— М.: 000 «И. Д. ВильямС», 2016. — С. 35.

9. Пинчук А. Использование методов Deep Learning в задаче выявления мошенничества // Банковское обозрение. Приложение "BEST PRACTICE". 2018. № 2. C. 30 -35.

10. Ференец В. DeepFake бросил вызов ЕБС // Банковское обозрение. 2020. № 1. С. 68-69.

11. П. 12 Постановления Пленума Верховного Суда РФ от 27.05.1998 № 9 «0 судебной практике по делам о преступлениях, связанных с наркотическими средствами, психотропными, сильнодействующими и ядовитыми веществами».

12. 228-я в граммах и сроках // "Коммерсантъ" от 18.06.2019, 16:20. [Электронный ресурс] https://www.kommersant.ru/doc/3999368 (дата обращения: 29.7.2020).

13. [Электронный ресурс] https://zakupki.gov.ru/epz/main/public/home.html (дата обращения: 29.7.2020).

14. [Электронный ресурс] http://www.ved.gov.ru/rus_export/resources/ (дата обращения: 29.7.2020).

15. Маклаков А. Г. Общая психология.—- ПП: Питер. - 2000.

16. Давыдов А.С., Квантовая механика. 2-е издание, М: Наука, 1973.

17. Busemeyer J.R., Bruza P. D. Quantum models of cognition and decision. - Cambridge University Press, 2012.

18. Haven E., Khrennikov A. Quantum Social Science. New York: Cambridge University Press, 2013.

19. Петренко В. Ф. Теоретические и методологические проблемы психологии: взаимосвязь квантовой физики и психологии сознания // Психологический журнал.— 2014.— - . 35.—N6.—C. 69-86.

20. Кругляков Э. П. Ученые с большой дороги // Комиссия по борьбе с лже- наукой РАН. — М.: Наука. — 2009.

21. Ilya A. Surov, Sergey V. Pilkevich, Alexander P. Alodjants and Sergey V. Khmelevsky, Quantum Phase Stability in Human Cognition// Frontiers in Psychology.V.10, p. 929. 2019. 
22. Кадомцев Б.Б., Динамика и информация. М.: Редакция журнала «Успехи физических наук», 1997.

23. Валиев К.А., Кокин А. А., Квантовые компьютеры: надежды и реальность. Ижевск: Научно-издательский центр «Регулярная и хаотическая динамика», 2000.

24. Platonov A.V., Bessmertny I. A., Semenenko E. K., Alodjants A. P. Non-separability “Effects in Cognitive Semantic Retrieving”. In: Aerts D., Khrennikov A., Melucci M., Toni B. (eds) Quantum-Like Models for Information Retrieval and Decision-Making. STEAM-H: Science, Technology, Engineering, Agriculture, Mathematics \& Health. Springer, 2019.

25. Bennett C.H. et al. Mixed-state entanglement and quantum error correction // Phys. Rev. A — At. Mol. Opt. Phys. 1996.

26. Melnikov A.A., Fedichkin L. E., Alodjants A. Predicting quantum advantage by quantum walk with convolutional neural networks // New Journal of Physics 2019, Vol. 21, No. 12, pp. 125002. https://doi.org/10.1088/1367-2630/ab5c5e

( Ю Юрьев Родион Николаевич ( juryev7@gmail.com ), Алоджанц Александр Павлович.

Журнал «Современная наука: актуальные проблемы теории и практики»

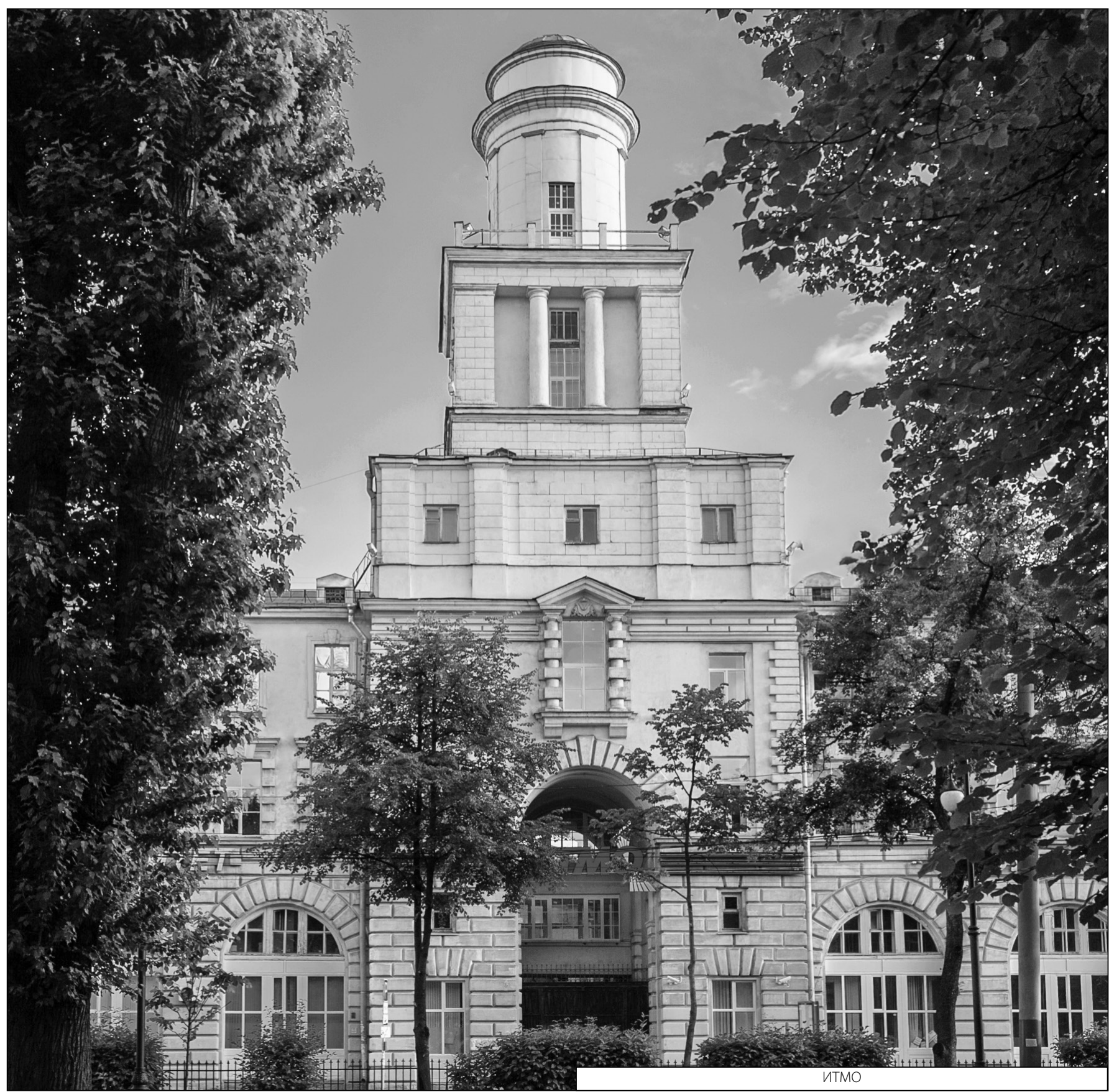

